Panorama EConómico, Volumen V, No. 9, julio-diciembre, 2009, pp. 97-135

\title{
EXTERNALIDADES DE RED Y SU INFLUENCIA EN LA PERCEPCIÓN DE MERCADO
}

\author{
Raúl Francisco Montalvo Corzo* \\ Ana Marcela Torres Hernández
}

\begin{abstract}
RESUMEN
Hoy en día los negocios están experimentando una nueva dinámica de expansión acompañada de una estrategia que conlleva un mayor posicionamiento ya sea de concepto, marca, producto o una combinación de éstos. Por medio de un modelo de localización y el uso de un juego en dos etapas, medimos ante diversos escenarios de competencia las externalidades generadas por los efectos de la presencia de una red de sucursales o puntos de venta, tanto para los consumidores como para las empresas, partiendo del hecho de que ambos buscan maximizar sus respectivas utilidades. Los resultados del modelo revelan que la cobertura del mercado sí tiene un efecto en variables como calidad percibida del producto, precio, y al mismo tiempo estas situaciones afectan el nivel de competencia ante la presencia de empresas rivales.

Palabras clave: Efectos de red, externalidades, cobertura de mercado, posicionamiento

Clasificación JEL: D62, L19, M21
\end{abstract}

* Los autores son profesores del Instituto Tecnológico de Monterrey, Campus Guadalajara. Correo electrónico: <rmontalvo@itesm.mx>. 
Raúl Francisco Montalvo Corzo/Ana Marcela Torres Hernández

\begin{abstract}
Nowadays, businesses have experienced a new dynamic of expansion together with the need of a positioning strategy that has to do with either the concept, the brand, the product or a combination of them. By using a location model and a two stage game we measure in several different competition scenarios the externalities generated by the effects of the presence of a network of branches or selling points, for both the consumers and the firms, taking into account that both look for the maximization of their utilities and profits respectively. The results from the model show that market coverage has an effect over variables such as perceived quality, price, utility and profits. And at the same time these situations affect the level of competition under the presence of rival firms.

Keywords: Network effects, externalities, market coverage, positioning JEL classification: D62, L19, M21
\end{abstract}

\title{
1. INTRODUCCIÓN
}

Desde la década de los noventas se han venido presentado, cada vez con más frecuencia, casos exitosos de empresas que han desarrollado un modelo de negocios basado en redes, el cual les ha permitido adueñarse de un alto porcentaje de participación de mercado1 dentro de sus respectivas categorías de producto. Una característica que tienen en común todas estas empresas, es la expansión constante y creciente de sus puntos de venta o sucursales. La cobertura de mercado, ${ }^{1}$ derivada de la extensión de la red de sus sucursales, ha sido un aspecto fundamental para lograr un posicionamiento privilegiado dentro de la mente de los consumidores. El desarrollo de negocios que han basado

\footnotetext{
${ }^{1}$ Entendiendo que su posicionamiento se debe al nivel de conocimiento y/o percepción en la mente de los consumidores sobre la empresa o su oferta.
} 
su crecimiento en la creación de redes, originaron el interés por buscar analizar de diferente forma el comportamiento de los mercados de redes principalmente en el sector consumo, ya que su estudio había sido mayormente enfocado a industrias relacionadas con la creación de conocimiento y tecnología.

Los aspectos económicos relacionados con las industrias de redes están basados en la existencia de externalidades positivas de red. Este concepto se fundamenta en la interdependencia de la función utilidad de un consumidor al comprar un producto con las decisiones de compra de otros consumidores. La literatura sobre externalidades de red advierte que, para que éstas se presenten es necesario cumplir algunas condiciones, tales como el hecho de que una empresa requiera alcanzar cierto nivel de "masa crítica", esto es, un mínimo requerido de personas que consumen un mismo producto, para generar un efecto positivo en la utilidad de los demás consumidores al ser parte de un conglomerado de clientes con posibilidad de interactuar entre sí, un ejemplo de ello es la telefonía celular. En este sentido, la formación de un reconocimiento de "marca" de las empresas puede jugar un papel importante al ser influenciado de manera positiva por la construcción de redes de consumidores lo suficientemente grandes como para comenzar a generar externalidades de red.

El objetivo principal de este artículo gira en torno al estudio de la relación que guarda el aumento de la cobertura geográfica ${ }^{2}$ de una empresa con la formación de redes de consumidores, así como el impacto que genera sobre las utilidades de las empresas la creación de estas redes.

La hipótesis central es que, al aumentar el número de sucursales de una marca, debido a la continua exposición a la que los consumidores están sujetos se genera un proceso continuo de fortalecimiento y posicionamiento de marca, de igual forma sustentado en el hecho de la posibilidad, bajo ciertas circunstan-

\footnotetext{
${ }^{2}$ Número de sucursales.
} 
cias, de que puede existir un aumento en el número de sus consumidores y, por lo tanto, de participación de mercado. En relación a esto, cuando una marca alcanza un posicionamiento efectivo su consumo confiere a los consumidores un valor subjetivo de status, ${ }^{3}$ puesto que sus productos son consumidos y reconocidos por otras personas que pertenecen a una misma red. Aunado a ello, en este estudio se presenta también un análisis del comportamiento de empresas en competencia bajo una estructura de duopolio, donde se supone la presencia de externalidades positivas de red en el consumo. Para ello se utiliza un modelo de localización de dos periodos. En el primer periodo los consumidores se forman expectativas sobre el tamaño de red de cada empresa en competencia; $\mathrm{y}$, en el segundo las empresas participan en un juego de precios, tomando como dadas las expectativas de los consumidores respecto al nivel de cobertura de cada empresa y del tamaño de la red. Partiendo del precio hedónico, ${ }^{4}$ formado tanto por el valor inherente al producto como por el valor derivado de la externalidad de red y de otras variables, se obtiene el nivel de cobertura de las empresas y, por último, resolviendo el modelo se obtienen las utilidades de equilibrio.

Adicionalmente, se analizan nueve posibles escenarios de competencia entre las dos empresas presentes en el mercado. Dichos escenarios se construyeron otorgando diferentes combinaciones de valores (bajo, medio y alto) a cada variable que conforma las utilidades de equilibrio de ambas empresas: calidad, valor de la externalidad de red, precios y costos. El análisis de los escenarios gira en torno a la relación que guardan las variables de equilibrio con las variables que conforman el precio hedónico. Es decir, se estudia bajo qué condiciones las variables ejercerán un efecto positivo o negativo sobre el nivel de cobertura y las utilidades generadas por las empresas.

\footnotetext{
3 Partiendo de que el "status" confiere un reconocimiento o deseo de pertenencia a un grupo y que su conceptualización puede tener más de una interpretación debido a una diversidad de condiciones socio-demográficas, por mencionar alguna.

4 Precio ajustado de los productos en función de los diferentes elementos de valor ofrecidos por las empresas (Katz y Shapiro, 1985).
} 


\section{REVISIÓN DE LITERATURA Y ANÁLISIS CONCEPTUAL}

La mayoría de la literatura existente de externalidades de red proviene predominantemente de la esfera de la organización industrial. Para Henard (1998), el razonamiento detrás de las externalidades de red consiste en que "un producto, que se vuelve ampliamente disponible y usado por más personas en un grupo de referencia (red), ${ }^{5}$ subsecuentemente se vuelve más valorado por cada individuo dentro de la red. Es un ciclo positivo de eventos, que se alimentan del continuo crecimiento y expansión de la red". Por lo tanto, la formación y expansión de las redes constituye una base para la creación de valor de un producto, tanto para los consumidores como para las empresas que lo ofrece. Asimismo, el autor establece que los principales temas estudiados en la literatura de externalidades de red pueden agruparse en las siguientes tres grandes vertientes: diseño de compatibilidad, patrones de adopción y ventajas de primeras adopciones. ${ }^{6}$

La literatura de externalidades de red puede dividirse en dos enfoques de estudio. El primer enfoque corresponde al análisis que gira en torno al supuesto de la existencia de externalidades de red y, bajo este supuesto, se intentan explicar las consecuencias de su existencia. El segundo enfoque, por otro lado, se centra en encontrar las causas que generan las externalidades de red (Economides, 1996).

Otra forma de clasificar los estudios desarrollados sobre externalidades de red, es con base en el tipo de análisis realizado, ya sea desde la perspectiva de la demanda o de la oferta. En los estudios correspondientes a la demanda el análisis gira en torno a los consumidores, los cuales deben anticipar cual tecnología (o producto) será mayormente utilizado por otros, debido a que se supone una función de utilidad interdependiente. Por otro lado, desde el

\footnotetext{
${ }^{5}$ Dentro de la cual existen individuos que guardan factores en común.

${ }^{6}$ Estas vertientes concentran el grueso de los estudios realizados sobre externalidades de red, sin embargo, no son exclusivos.
} 
punto de vista de la oferta el análisis gira en torno a la forma en que las tecnologías son elegidas o promovidas por las empresas (Tirole, 2000).

Las industrias que han sido analizadas bajo estos enfoques son aquellas que dependen intensamente de la creación de tecnología y de conocimiento. Es por esto que en la literatura de externalidades de red se utiliza ampliamente el concepto de "tecnología" dominante, que podría también definirse como "producto" dominante, al estudiar aquellas industrias cuya actividad no esté relacionada principalmente con la creación de tecnología.

En los trabajos centrados en el diseño de compatibilidad se estudian, ya sea la compatibilidad de productos de una misma empresa (intra-firma) o la compatibilidad de productos entre empresas competidoras en el mercado (intertecnológica). Idealmente, las firmas preferirán la existencia de una sola red para optimizar sus utilidades, sin embargo en la realidad más de una red puede existir. Bajo este esquema, la finalidad de las empresas será tratar de convertirse en el "estándar"7 del mercado y de esta manera poder crear una red única, de la cual obtendrán todas las ganancias.

Siguiendo esta línea, Liebowitz y Margolis (1994) mencionan que, algunas propuestas de modelos de externalidades de red predicen que a medida que tecnologías nuevas se posicionan en la economía, la participación de la economía caracterizada por retornos crecientes aumentará. ${ }^{8}$

En este artículo utilizaremos el término de "empresa" al referirnos a un agente económico que produce y ofrece sus productos a los consumidores. Por simplificación, suponemos que cada empresa maneja una sola marca, entendida como el concepto mediante el cual los consumidores identifican a la empresa, a sus productos y a sus establecimientos.

Un interesante análisis orientado a externalidades de red analiza los efectos de la disminución de la demanda de un bien, proveniente de un mayor nú-

\footnotetext{
${ }^{7}$ Es decir, el más utilizado por los consumidores.

${ }^{8}$ El estudio sobre la relación entre la presencia de externalidades de red y retornos crecientes queda fuera del alcance del presente trabajo.
} 
mero de personas que consumen ese bien. Este comportamiento obedece al deseo de exclusividad de las personas, de diferenciarse de "la manada", concepto definido como snob, de acuerdo a Liebenstein (1950). En su trabajo el autor atribuye el nacimiento de los efectos de red a la "disciplina de la mercadotecnia, donde se entiende que el éxito de un producto o servicio es un fenómeno que se fortalece a sí mismo" y denomina este fenómeno como el efecto bandwagon, o "arrastre", que consiste "en la medida en la cual la demanda por un bien se incrementa debido al hecho de que otros también están consumiendo el mismo bien... Representa el deseo de las personas por comprar un bien, con la finalidad de igualar a las personas con las que desean ser asociadas".

Sin embargo, formalmente el concepto de "externalidades de red" surge del trabajo de Katz y Shapiro (1985), donde los autores consideran "la existencia de externalidades positivas de red en el consumo cuando la utilidad de un consumidor derivada por el consumo de un bien incrementa con el número de otros consumidores que también consumen el mismo bien". Adicionalmente, mencionan también la existencia de otras posibles causas más sutiles de externalidades de red, como son: "i) el hecho de que el acceso a la información de las marcas populares esté más disponible para los consumidores; ii) la marca que es líder en la participación de mercado es sinónimo de calidad del producto para los consumidores; y iii) efectos puramente psicológicos de seguir 'la moda' o querer consumir un producto porque la mayoría lo consume".

De acuerdo con Bensen (1999), existen básicamente dos causas que generan externalidades de red en el consumo. Por un lado, las externalidades de red directas, que surgen cuando un incremento en el tamaño de la red incrementa el número de otros consumidores que consumen el mismo producto. Y, por otro lado, las externalidades de red indirectas, que surgen cuando un incremento en el tamaño de una red expande el rango de productos complementarios disponibles para los miembros de la red.

Como podemos observar, todas estas causas están relacionadas de una forma u otra con el nivel o posicionamiento de marca de una empresa. Con 
base a esto, podemos inferir que existe una relación positiva entre el reconocimiento de marca y la posibilidad de generar externalidades de red.

Un aspecto clave del análisis de externalidades de red se refiere al concepto "masa crítica" o base instalada, es decir, la cantidad mínima de personas que consuman un mismo producto necesarias para comenzar a generar externalidades de red. En este sentido, Cáliz (1985) (citado en Xie y Sirbu, 1995) menciona que "a medida que un producto es introducido, existe una incertidumbre asociada con la experiencia sobre los atributos de los productos. El valor de un producto nuevo para un consumidor adverso al riesgo es menor que el valor del producto, si toda la información de experiencia está disponible. Mientras más consumidores adopten la innovación, más de la incertidumbre relacionada se elimina y la valoración del producto se incrementa". Por lo tanto, alcanzar el nivel requerido de "masa crítica" es una condición necesaria para comenzar a generar externalidades de red. En este sentido, si una empresa comienza a expandir su red de sucursales antes de alcanzar el nivel necesario de "masa crítica, no será capaz de generar externalidades positivas de red en el consumo.

Otro concepto fundamental en la teoría de externalidades de red es el "punto de congestión", que se refiere al nivel alcanzado por una red después del cual la adición de un consumidor marginal a ésta, en lugar de generar un efecto positivo sobre la utilidad de los demás consumidores, generará una desutilidad. Por lo tanto, el tamaño de la red no puede tener un crecimiento indefinido, existe un límite en cual las externalidades de red dejarán de generar beneficios adicionales al consumo de los productos y después del cual comenzarán a generar costos. ${ }^{9}$

Otro aspecto importante sobre las externalidades de red se refiere a que, el análisis de las mismas tiene su fundamento en las "expectativas" de los consumidores sobre el número sucursales que esperan una empresa tenga y,

\footnotetext{
${ }^{9}$ En los que pudieran llegar a incurrir los consumidores al congestionarse la red: tiempo de espera, saturación, falta de servicio o mala calidad del mismo.
} 
por lo tanto, del número de consumidores que se espera consumirán la marca. Específicamente, los consumidores se forman expectativas sobre el tamaño que tendrán las redes de las empresas en competencia y sus decisiones de compra estarán basadas en ellas. "En los mercados de redes no es el nivel actual de ventas o de ventas acumuladas lo que determinará el ganador, ${ }^{10}$ en su lugar, las expectativas sobre el tamaño final de la red son cruciales" (Bensen y Farrell, 1994). Por último, es importante citar una reflexión de éstos autores en referencia a los efectos ocasionados en los mercados donde existe la presencia de externalidades de red: “...el interés de estudiar las externalidades de red, radica en que su existencia genera cambios significativos en la manera en que se desarrolla la competencia en los mercados donde están presentes. Las características de los mercados de redes, producen que la competencia entre productos no sea sólo una cuestión de productos ligeramente mejores, o con costos ligeramente menores, y de esta manera, la obtención de beneficios ligeramente mayores. En su lugar, pequeñas diferencias, ya sea en percepción o realidad, pueden ser magnificadas en un proceso en el cual algunas empresas hacen ganancias extremadamente grandes, y en donde las posiciones de mercado dominantes son difíciles de cambiar. Cuando los compradores esperan obtener beneficios de red de uno de los productos de una empresa que otras empresas no pueden proveer, se crea una discrepancia grande en el valor [del producto], de la cual la empresa afortunada puede ser capaz de extraer ganancias".

En el trabajo de Economides y Flyer (1997) se analizan las economías de industrias en donde las externalidades de red son significativas. Los autores asumen que, en tales industrias las empresas tienen altos incentivos de adherirse a estándares de compatibilidad técnica, de tal manera que se puedan cosechar externalidades de red a nivel industria. Sin embargo, también una

\footnotetext{
${ }^{10}$ Líder de mercado.
} 
empresa puede beneficiarse de producir un producto incompatible. En este trabajo, los autores muestran cómo la competencia equilibra estos incentivos opuestos.

Bental y Spiegel (1995) por su parte, presentan un modelo en el cual identifican la calidad de un producto de redes mediante el número de consumidores que lo utilizan, de tal manera que los productores no podrán establecer unilateralmente la calidad del producto y ésta no está relacionada únicamente con las características físicas del mismo. En este trabajo los autores estudian la cobertura de mercado bajo diversas estructuras de la industria, encontrando que en las industrias no cooperativas ${ }^{11}$ resulta una cobertura de mercado más grande que en las cooperativas. ${ }^{12}$ Por otro lado, si existe libre entrada de productores al mercado, la cobertura de mercado con redes no compatibles será la más grande. Y, por último, si no existe libre entrada al mercado, la cobertura será mayor en industrias con un sólo estándar.

Katz y Shapiro (1992) consideran que las ventajas de la primera empresa en establecer un estándar representan una barrera de entrada muy fuerte, inclusive cuando la tecnología retadora sea claramente superior. Particularmente, en su modelo se considera la existencia de exceso de inercia y de exceso de velocidad en el proceso de adopción de una nueva tecnología. Adicionalmente, analizan los incentivos de las empresas para hacer sus productos compatibles y concluyen que la empresa que introduce la nueva tecnología tiene una predisposición negativa hacia la compatibilidad. En dicho trabajo se exploran también las consecuencias en el bienestar social, ante lo cual los autores concluyen que, la empresa que propone la nueva tecnología gana mayores beneficios de los que su entrada contribuye al bienestar social.

Por otro lado, Farrell y Saloner (1986) examinan si los beneficios de la estandarización pueden llevar a una industria a estancarse en un estándar obsoleto e inferior. Los autores concluyen que pueden presentarse tanto inercia

\footnotetext{
${ }^{11}$ Aquellas de estructuras competitivas.

${ }^{12}$ Las de estructura de "cártel".
} 
excesiva como velocidad excesiva, y que estos problemas pueden solucionarse por medio de estrategias de comunicación.

En Saloner y Shepard (1995), se realiza una aplicación empírica del proceso de adopción de la tecnología de cajeros automáticos (ATM) durante la década de los setentas. En este trabajo los autores proponen un modelo en el cual se estudia de manera independiente el "efecto red", entendido como el número de sucursales que adoptan la tecnología de cajeros automáticos, y el "efecto producción de escala", que se refiere al aumento en el número de usuarios de la tecnología. El principal descubrimiento que se realiza en este estudio es que, los bancos con mayor número de sucursales adoptan la tecnología ATM antes que aquellos bancos con pocas sucursales. Este resultado es consistente con la presencia de los "efectos de red". Dado esto, los beneficios de los consumidores se incrementan a medida que incrementa el número de personas en el sistema. Este último efecto es la causa de las externalidades de red, porque cada usuario nuevo confiere una utilidad adicional a los demás usuarios.

Desde el punto de vista de la Mercadotecnia existen también una serie de trabajos que refuerzan la teoría de efectos de red. Como se comentó anteriormente, un exitoso modelo de negocios relacionado con la formación de redes se ha venido desarrollando y consolidando en los últimos años. Este modelo tiene sus fundamentos en la construcción de redes de consumidores, mediante la creación de percepciones y expectativas en los consumidores sobre las marcas presentes en el mercado, en cuanto a su calidad, nivel de cobertura y de número de clientes. Generalmente, el modelo de negocios de redes ha proporcionado resultados sumamente positivos sobre las utilidades de las empresas que lo han seguido. Una explicación a estos resultados puede atribuirse al alto grado de posicionamiento que han logrado alcanzar por medio de la formación de redes.

Dentro del presente contexto de competencia de mercados, cada vez más las empresas necesitan lograr que se grabe una distinción en la mente de los consumidores sobre los productos que ofrecen. Ante la confusión que puede generar el exceso de opciones sobre distintos productos parecidos presentes en el mercado, aunado a la inseguridad que acompaña la toma de decisiones en esas circunstancias, las marcas se convierten en un elemento de confiabilidad. 
Es decir, "por los atributos y valores que tengan los productos, las marcas son depositarias del posicionamiento" (Homs, 2003). Por lo tanto, podemos decir que "el posicionamiento de una marca implica implantar los beneficios distintivos y la diferenciación de la marca en la mente de los consumidores" (Kotler y Armstrong, 2003).

Es importante mencionar que la fuerza de una marca puede ser analizada desde dos perspectivas: los actos del consumidor y las estrategias de la compañía (Davis, 2002).

Desde el punto de vista de las empresas, para alcanzar un posicionamiento éstas tratan de implementar diversas estrategias, ${ }^{13}$ con la finalidad de establecer un concepto con el cual desean ser relacionados en la mente de los consumidores. Para que las estrategias emprendidas por una empresa tengan éxito y se genere un valor "de marca", los consumidores deben estar convencidos de que existen diferencias significativas entre las distintas empresas que ofrecen sus productos dentro de una misma categoría (Kotler y Keller, 2006).

El comportamiento de los negocios basados en redes ha generado un nuevo funcionamiento de la economía. Podemos considerar que el crecimiento de la nueva economía está regido por ciclos de autoconsolidación. Consideremos la ley de Metcalfe, en donde se establece que "el coste de la red se expande proporcionalmente con el incremento en el tamaño de la red, pero el valor de ésta aumenta exponencialmente" (Kotler, Jain y Maescinsee, 2002).

\section{EJEMPLOS DE MODELOS DE NEGOCIOS DE REDES EN LA ACTUALIDAD}

Uno de los ejemplos más representativos del modelo de negocio de redes en la industria de consumo es la cadena minorista de cafeterías Starbucks. Esta

\footnotetext{
${ }^{13}$ Por ejemplo, calidad en el producto y/o servicios ofrecidos, servicios post-compra, horarios de 24 horas, servicio personalizado, instalaciones confortables o adaptadas para proporcionar una sensación de seguridad, exclusividad, mayor cobertura de mercado, etc.
} 
empresa ha aumentado el número de sus cafeterías de una manera impresionante; en promedio, durante el año 2006 se inauguraron 6 cafeterías por día en todo el mundo. Esta expansión ha sido acompañada de un crecimiento constante en sus ingresos. En el año 2002, Starbucks tenía instaladas 4709 sucursales y en el 2007 ya contaba con más de 15000 en 42 países. Al mismo tiempo, los ingresos netos de la empresa en el mismo rango de años tuvieron un crecimiento acumulado del 29\%, pasando de 210 a 673 millones de dólares. En relación al éxito extraordinario de la empresa, Philip Kotler, en uno de sus textos, expresa su opinión sobre el origen del éxito de la compañía: “¿Cómo puede mantener Starbucks su fenomenal tasa de crecimiento donde las ganancias son del 40 por ciento?, la compañía ha decidido que el mejor camino es la expansión" (Kotler y Armstrong, 2003). A pesar del aumento en los canales de distribución de los productos que Starbucks ofrece, en el año 2007 el grueso de sus ingresos, específicamente el 85 por ciento, provinieron de las ventas realizadas en sus sucursales. ${ }^{14}$

A pesar de esta expansión avasalladora, los directivos de la compañía consideran que "aún existen oportunidades significativas de desarrollo de tiendas tanto doméstica como internacionalmente". ${ }^{15}$ Después de 5 años de su llegada a México, en el 2007 Starbucks contaba ya con 159 establecimientos, ${ }^{16}$ y su tasa de expansión correspondió a la apertura de una cafetería cada tercer día. Su objetivo en el mercado mexicano, aún no termina, ya que tienen esperado establecer entre 800 y 1500 locaciones $^{17}$ en este país.

Otro ejemplo relevante de este tipo de negocios se refiere a la cadena de comida rápida McDonald's. Esta empresa contó durante el 2007 con más de 31000 sucursales en 118 países alrededor del mundo, generando durante ese mismo año ingresos netos de 2395 millones de dólares, reflejando en estos resultados un crecimiento constante que esta empresa ha experimentado en

\footnotetext{
${ }^{14}$ Starbucks, Fiscal 2007 Annual Report.

15 Starbucks Coffee Company Fiscal 2007, Third Quarter Financial Results Prepared Remarks.

${ }^{16}$ Starbucks Corporation, 10-K Report, Fiscal 2007.

17 Ángeles, 2007.
} 
sus utilidades, comparándolos con los ingresos netos del 2002, los cuales alcanzaban los 893 millones de dólares. ${ }^{18}$

Este fenómeno de expansión y cobertura internacional también está presente en las cadenas minoristas de ropa, tal es el caso de la marca de ropa Zara, perteneciente al grupo INDITEX. Este corporativo español maneja 8 marcas de ropa, siendo Zara su marca más valiosa. En el año 2007 las tiendas Zara alcanzaban 1361 sucursales en 68 países alrededor del mundo, añadiendo a su cadena tan sólo durante dicho año 186 tiendas más. En ese mismo año, la cadena presentó un crecimiento del $23 \%$ de su utilidad operativa respecto al año anterior. ${ }^{19}$ En México, la cadena contaba con 47 tiendas establecidas a lo largo del país.

Continuando con los ejemplos de cadenas minoristas internacionales de ropa, otro caso exitoso se refiere a la empresa $C \& A$, que cuenta con presencia en 20 países de Europa, América y Asia. El total de tiendas localizadas alrededor del mundo en el 2007 superaban las 1000 sucursales. En México, en el mismo año contaban con 55 sucursales localizadas en 22 ciudades diferentes, representando un crecimiento considerable desde su primera inauguración en la Ciudad de Puebla en 1999. Los planes de expansión de la empresa en México continúan, ya que se espera se inauguren 5 tiendas más por año.

Al igual que en los ejemplos antes mencionados, el modelo de negocios de red también ha estado presente en el sector bancario. Tal es el caso de dos de los más importantes corporativos bancarios a nivel mundial: Grupo Santander y $H S B C$. Estos corporativos utilizan en su publicidad y mercadotecnia la cobertura de mercado con la que cuentan, ${ }^{20}$ perfilándose como uno de sus principales atractivos para los consumidores. Por su parte, Grupo Santander cuenta con presencia geográfica en más de 40 países de Europa Continental, el Reino Unido e Iberoamérica. En el año 2007 su red de establecimientos estuvo compuesta por más de 10800 sucursales, formando así la mayor red de

\footnotetext{
${ }^{18}$ McDonald's Corporation 2007 Annual Report.

${ }^{19}$ Inditex, Reporte Anual 2007.

${ }^{20}$ Derivada de su amplia red de sucursales minoristas ubicadas alrededor del mundo.
} 
oficinas minoristas en el mundo Occidental. Las utilidades generadas por Grupo Santander en ese año fueron de 8.11 millones de euros, obteniendo un crecimiento del $19.3 \%$ respecto al año anterior. ${ }^{21}$

Por su parte, desde el año 2002 HSBC estableció como su eslogan a nivel mundial la frase El banco local del mundo, en la cual se resalta la importancia de su cobertura mundial, considerándola como una de sus principales estrategias y fortalezas competitivas. Durante el 2007, su red de oficinas minoristas contaba con más de 10000 sucursales ubicadas 83 países. Durante el mismo año, el grupo financiero $H S B C$ alcanzó 87601 millones de dólares de ingresos operativos, creciendo un $25 \%$ respecto al año anterior. ${ }^{22}$

En México existen dos ejemplos muy claros de empresas que han adoptado como estrategia de negocio la expansión del número de sus sucursales, obteniendo excelentes resultados en sus utilidades. El primer caso, es el de las tiendas de conveniencia $O X X O$, empresa perteneciente al grupo FEMSA. ${ }^{23}$ En 1998 la red de establecimientos de $O X X O$ estaba compuesta por 1000 sucursales. Nueve años después es la cadena de tiendas de conveniencia más grande en América Latina con más de 5563 sucursales; su cobertura de mercado abarca 50 ciudades de 29 estados de México. En el 2007, en promedio se abrieron cada día dos nuevos establecimientos. En el mismo año, la cadena de tiendas $O X X O$ tuvo un crecimiento en el número sucursales del $14.8 \%$, y el crecimiento de su utilidad de operación fue del $39.1 \%$ respecto al año anterior. ${ }^{24}$

El segundo ejemplo es el de Farmacias Guadalajara. En el 2007, esta empresa ya contaba con 551 sucursales y su estrategia de expansión consistió en inaugurar, en promedio, una sucursal cada 5 días, abriendo únicamente durante este año 73 nuevas sucursales. Su red de sucursales abarca 144 ciuda-

${ }^{21}$ Grupo Santander, Informe Anual 2007.

${ }^{22}$ HSBC Holding pcl, 2007 Annual Report and Accounts.

${ }^{23}$ Grupo empresarial mexicano, cuyo principal negocio, además de la cadena OXXO, son la Cervecería Cuauhtémoc Moctezuma y Coca Cola FEMSA.

${ }^{24}$ FEMSA, Informe Anual 2007. 
des en 19 de los 32 estados de México. Las utilidades netas generadas por esta empresa durante el 2007 tuvieron un crecimiento del $21.5 \%$ respecto al año anterior. ${ }^{25}$

Una vez presentados sólo algunos ejemplos de empresas que han seguido la estrategia de negocios de creación de redes, se expondrá el modelo de localización de dos etapas, mediante el cual obtendremos el nivel de cobertura y utilidades de equilibrio que analizaremos bajo diversos escenarios de competencia para las dos empresas presentes en el mercado.

\section{EL MODELO}

Considerando la existencia de externalidades positivas de red en el consumo, se presenta un modelo de localización basado en el modelo expuesto por Montalvo (2003). En nuestro modelo consideramos dos empresas dentro de una misma categoría de producto que compiten por los consumidores presentes en el mercado. Suponemos al mercado como una línea recta con densidad igual a uno, el cual se reparte entre las dos empresas en competencia por medio de las decisiones de los consumidores, las cuales se basan en los valores de las variables que conforman el precio hedónico de cada una de las empresas.

Adicionalmente, tomando los fundamentos del modelo de Katz y Shapiro (1985) sobre externalidades de red, suponemos que no existe efecto ingreso y que los consumidores maximizan su utilidad para realizar sus decisiones de compra. Suponemos que los consumidores tienen información perfecta sobre el número de sucursales de las empresas. Asumimos también que la función de utilidad de los consumidores, derivada del consumo de un producto, es interdependiente a las decisiones de consumo de otros consumidores. ${ }^{26}$ De tal

${ }^{25}$ Corporativo Fragua, Informe Anual 2007.

${ }^{26}$ Este supuesto engloba la esencia de las externalidades de red. 
manera que la utilidad de un consumidor por la compra de un bien estará en función de las expectativas generadas sobre el tamaño de las redes de consumidores de las marcas presentes en el mercado.

Cada consumidor comprará una unidad o ninguna unidad de producto de la marca de su elección. Suponemos que las decisiones de compra de los consumidores se hacen antes de que se conozca el tamaño real de las redes. Suponemos también que en equilibrio las expectativas de los consumidores se cumplen. ${ }^{27}$

El modelo se desarrolla en un juego de dos etapas: en una primera etapa, los consumidores forman sus expectativas sobre el tamaño de red de cada marca, basándose en el número de sucursales con las que cuenta y con las que cree que contará. En la segunda etapa, las empresas, tomando como dadas las expectativas de los consumidores, asumen su rol en el mercado y cada una establece su precio. Basados en estos precios, los consumidores entonces realizan su elección de consumo, comparando los precios establecidos por las empresas con sus precios hedónicos, los cuales a su vez están en función de $i=1,2, \ldots$, Nos tamaños esperados de redes y de otras variables de valor.

Para simplificar el análisis asumimos que las empresas, al decidir establecer una nueva sucursal, realizan un riguroso estudio de mercado y de factibilidad. La decisión es tomada en función de la localización geográfica y de la cobertura de mercado que tendrá cada sucursal en particular. Es decir, las empresas fijan sus políticas o criterios de localización de sucursales con base en la población esperada ${ }^{28}$ de un área geográfica específica, que representa una medida estándar o promedio de la cantidad de posibles consumidores de la marca.

Llamaremos $x_{i}$ al número de sucursales o puntos de venta que tiene una empresa $i$, donde . Cada una de las sucursales de la empresa

\footnotetext{
${ }^{27}$ En el sentido de que si los consumidores esperan que una marca sea dominante, en cuanto al nivel de participación de mercado en su categoría de producto, ésta de hecho lo será. De igual manera, se refiere a que el tamaño de la red, en equilibrio, será igual al tamaño esperado de la misma.

${ }^{28}$ Entendiendo como población esperada aquella que habita en un área geográfica, y también aquella población que, sin habitar en esa zona, se estima pasará por ahí en sus traslados.
} 
genera su propio grupo de consumidores, es decir, forma su propia red alrededor de una misma marca.

Suponemos que cada sucursal tiene un tamaño exógeno de red, el cual, por simplificación, será igual para todas las sucursales, basándonos en el hecho de que las empresas manejan una política estándar de apertura y de cierre de sucursales. Se entiende que, en un sentido estricto, el tamaño de sucursal $z_{i}$ cae dentro de un rango de valores: ${ }^{29}$

$$
z_{i}=\left[\frac{\underset{\max }{z+z}}{2}\right]
$$

donde el límite inferior $\underset{\min }{z}$ representa el mínimo de consumidores requeridos para que una sucursal mantenga sus operaciones en el mercado; y el límite superior representa el número máximo de consumidores que, por su localización y tamaño, una sucursal puede atender sin que su incorporación signifique una disminución en la cantidad de consumidores de otra sucursal por debajo del límite inferior $\underset{\min }{z}$, y/o en la calidad ofrecida. Es decir, dicho intervalo representa la política de permanencia de las sucursales.

Nombramos a la expectativa que los consumidores se forman sobre el número de sucursales, el cual a su vez define el tamaño de red de la empresa $i .^{30}$ Esto es, puede ser definida como el tamaño percibido o esperado de la red $;^{31}$ en este sentido tenemos que

${ }^{29}$ Considerando que cada consumidor consume en promedio una unidad de producto de la marca de su elección, podemos decir que representa también el consumo promedio esperado de una sucursal.

${ }^{30}$ Las cuales, juntas o por separado, generan las expectativas de los consumidores en torno a la empresa y a la marca relacionada.

${ }^{31}$ Recordemos que los consumidores generan sus expectativas con base en el rango de valores de , del tamaño de red de las sucursales. 
donde $x_{i}$ representa el número de sucursales que tiene la empresa $i$.

En la medida en que un mayor número de consumidores conozca y consuma una marca, ésta irá alcanzando un posicionamiento más elevado ${ }^{32}$ hasta llegar al punto en el cual el consumo de sus productos generará un status. Debido a que la variable $x_{i}^{e}$ representa la medida del tamaño de red esperado de una marca, también representará una aproximación del nivel de status que proporciona su consumo. ${ }^{33}$ Suponemos que, en equilibrio las expectativas de los consumidores se cumplen.

Suponemos también que, las marcas que cuentan con una o más sucursales ofrecen productos, calidad y servicio homogéneos, es decir, manejan un mismo concepto. Por simplificación, asumimos que todas las sucursales de la empresa $i$ mantienen precios homogéneos, es decir, no realizan discriminación de precios entre sus sucursales.

$\frac{d x_{i=}^{e}}{d x_{i}} z_{i} x_{i}$

Podemos definir la relación como "efecto sucursal", es decir, el impacto que genera el número de sucursales sobre el conocimiento y la consiguiente percepción de la marca. Dado que el "efecto sucursal" es positivo, > 0 , el crecimiento en el número de sucursales impactará positivamente el tamaño de red, añadiendo consumidores más por cada sucursal nueva que se agregue.

Suponemos que los consumidores son heterogéneos en cuanto a su disposición mínima de pago por una marca. Es decir, existen distintos tipos de consumidores que difieren en su disposición mínima de pago por la misma marca.

\footnotetext{
32 A través del valor de la red, que podemos considerar como una aproximación del posicionamiento de marca.

${ }^{33}$ Existen productos que tienen el efecto inverso, específicamente aquellos que buscan proporcionar una sensación de "exclusividad". Es decir, a medida que un mayor número de personas los consuma, el valor que les proporcionará disminuirá, ya que pierden su esencia y la finalidad para la que fueron creados.
} 
Específicamente, tenemos que un consumidor de tipo $r$ estará dispuesto a pagar $r+q_{i}+v\left(x_{i}^{e}\right)$ por una marca, para la cual el tamaño de red esperado es $x_{i}^{e}$ (Katz y Shapiro, 1985). La variable $r$ representa el valor inherente que el consumidor asigna al producto en sí mismo y a todos aquellos atributos de una marca que buscan satisfacer sus necesidades y gustos; ${ }^{34}$ esto es, $r$ representa el precio de reserva del consumidor. La variable $q_{i}$ representará la calidad estándar del producto en cuanto a sus propiedades físicas reales y no las percibidas por los consumidores. Mientras mayor sea el número de características deseables con las que cumpla la marca, mayor será la disposición mínima de pago de un consumidor por sus productos.

Por último, la función , donde $\delta=$ factor exógeno de valoración de la red, representa el valor que el consumidor asigna a la externalidad de red, o al status generado por el posicionamiento del que goza la marca en el mercado. Suponemos que la función $v\left(x_{i}^{e}\right)$ es continua y dos veces diferenciable, tal que, $v^{\prime}>0, v^{\prime \prime}<0$ y $\lim v^{\prime}(x)=0$ a medida que $x \rightarrow \infty .{ }^{35}$

Normalizando $r$ y $v(0)$ tenemos que $v(0)=0$. Asumimos que $r$ tiene densidad 1 y está uniformemente distribuida entre $-\infty$ y $A,{ }^{36}$ es decir, $r \sim[-\infty, A]$; asumimos también que $A>0 .{ }^{37}$

Suponemos la existencia de un costo de transporte por unidad de distancia $t$, en el que incurren los consumidores al tener que trasladarse a una de las sucursales de la empresa para acceder a sus productos. ${ }^{38}$

\footnotetext{
${ }^{34}$ Por ejemplo, un servicio amable, un ambiente confortable, amplios horarios, instalaciones iluminadas, etc.

35 Tales características de la función obedecen al concepto "punto de congestión" relacionado con las externalidades de red.

${ }^{36}$ Donde $A$ representa el precio máximo de reserva.

${ }^{37}$ El supuesto de densidad uniforme implica suponer que el producto tiene una curva de demanda lineal. Asumimos que los valores de $r$ no tienen un límite finito inferior, para evitar considerar soluciones de esquina, donde todos los consumidores entran al mercado (Katz y Shapiro, 1985).

${ }^{38}$ El valor de esta variable estará inversamente relacionado con el número de sucursales de la empresa.
} 
Cada consumidor elegirá consumir la marca que maximice su utilidad con base en las variables antes mencionadas. Es decir, los consumidores se enfrentan a un proceso de autoselección entre una gama de alternativas existentes en el mercado para una misma categoría de producto. Este proceso se desarrolla de la siguiente manera: sea $p_{i}$ el precio establecido por la marca $i$ en el mercado, un consumidor de tipo $r$ escogerá la marca que maximice el valor $\mathrm{de}^{39}$

$$
r+q_{i}+v\left(x_{i}^{e}\right)-\lambda t-p_{i}
$$

La decisión de compra de los consumidores está condicionada a la siguiente relación ${ }^{40}$

$$
r+q_{i}+v\left(x_{i}^{e}\right)-\lambda t-p_{i}>0
$$

La elección de consumir una marca dependerá del valor que ofrece la empresa al consumidor, $\left(r+q_{i}+v\left(x_{i}^{e}\right)\right)$, así como del precio que cobra por sus productos $\left(p_{i}\right)$ y del costo por acceder a ellos $(\lambda t)$. Para que un consumidor decida consumir una marca, los tres componentes de valor ofrecidos por la empresa, $r+q_{i}+v\left(x_{i}^{e}\right)$, tienen que ser necesariamente mayores al costo total de consumo (precio del producto + el costo de acceso al mismo).

Para que dos o más empresas generen ventas positivas, tiene que cumplirse la siguiente relación:

$$
p_{i}+\lambda t-v\left(x_{i}^{e}\right)-r-q_{i}=p_{\neg i}+(1-\lambda) t-v\left(x_{\neg i}^{e}\right)-r-q_{\neg i}
$$

\footnotetext{
${ }^{39}$ El grupo de consumidores que conforman los distintos valores de $r$ representan un mismo segmento, por lo tanto, su disposición mínima de pago es igual y está representada por los distintos valores de $r$.

${ }^{40}$ Cuando para un tipo de consumidor $r$, (3) es negativa para todas las $i$, entonces el consumidor decide no consumir ninguna marca y, por lo tanto, no entra al mercado.
} 
Por lo tanto, $p_{i}+\lambda t-v\left(x_{i}^{e}\right)-r-q_{i}$ representa el precio hedónico para la marca $i$. Llamaremos $\lambda$ y $1-\lambda$ a las variables que representan la cobertura y/ o participación de mercado de las empresas $i$ y $\neg i$ respectivamente, las cuales están uniformemente distribuidas entre 0 y 1 , debido a que suponemos al mercado total como una línea con densidad 1 . Las variables de $\lambda$ y $1-\lambda$ están determinadas por los valores de los componentes del precio hedónico, como se mostrará más adelante.

Por lo tanto, un consumidor maximizará su utilidad en función de su decisión de consumo entre dos marcas, considerando el valor de su respectiva participación de mercado.

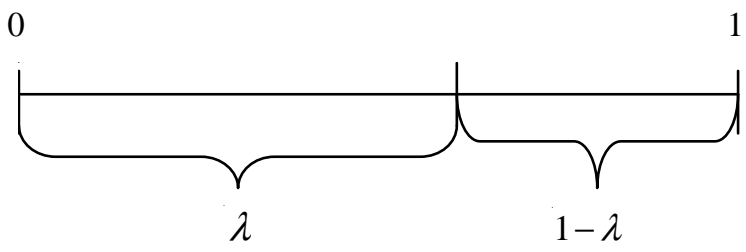

Definimos $a$ y $b$ como:

$$
a=r+q_{i}+v\left(x_{i}^{e}\right)-\lambda t-p_{i}
$$

$\mathrm{y}$

$$
b=r+q_{\neg i}+v\left(x_{\neg i}^{e}\right)-(1-\lambda) t-p_{\neg i}
$$

Con base en lo anterior podemos obtener las siguientes conclusiones:

a) Cuando $a=b$ un consumidor será indiferente entre consumir la marca $i$ y la marca $\neg i$, ya que el consumo de cualquiera de las dos le genera la misma utilidad;

b) Un consumidor preferirá la marca $i$ cuando $a>b$; y

c) Un consumidor preferirá la marca $\neg i$ cuando $b>a$. 
Una vez dicho lo anterior procedemos a resolver para $\lambda$, partiendo de la siguiente ecuación:

$$
r+q_{i}+v\left(x_{i}^{e}\right)-\lambda t-p_{i}=r+q_{\neg i}+v\left(x_{\neg i}^{e}\right)-(1-\lambda) t-p_{\neg i}
$$

Partiendo de (8), obtenemos que el valor de $\lambda$ es:

$$
\lambda=\frac{q_{i}-q_{\neg i}-p_{i}+p_{\neg i}+v\left(x_{i}^{e}\right)-v\left(x_{\neg i}^{e}\right)+t}{2 t}
$$

Por otro lado, el valor de $1-\lambda$ es el siguiente:

$$
1-\lambda=\frac{q_{\neg i}-q_{i}-p_{\neg i}+p_{i}+v\left(x_{\neg i}^{e}\right)-v\left(x_{i}^{e}\right)+t}{2 t}
$$

Los valores de $\lambda$ y $1-\lambda$ de participación de mercado, presentados en (9) y (10), se interpretan como un porcentaje del total del mercado, equivalente al número de individuos que compran esas marcas. Es decir, las consideramos una medida de la demanda de los productos de cada empresa. Recordemos que uno de nuestros supuestos se refiere a que cada consumidor consume en promedio una unidad de producto $y$, dado que en equilibrio los mercados se vacían, entonces (9) y (10) también pueden interpretarse como una medida de la producción de las empresas.

Por lo tanto, tenemos que las utilidades resultantes para cada una de las empresas son:

para la empresa $i$,

$$
\pi_{i}=\lambda\left[p_{i}-c_{i}\right]
$$


para la empresa $\neg i$.

$$
\pi_{\neg i}=(1-\lambda)\left[p_{\neg i}-c_{\neg i}\right]
$$

En (11) y (12), $c_{i}$ y $c_{\neg i}$ representan los costos totales ${ }^{41}$ de producción y de operación que pueden verse determinados por la densidad de su cobertura. No es ilógico pensar que cada empresa puede incurrir en costos distintos, esto es $c_{i} \neq c_{\neg i}$, aunque ésta no representa una condición necesaria.

Partiendo de las funciones de utilidad, las empresas emprenden un proceso de maximización para obtener los precios de equilibrio del mercado así como las utilidades de equilibrio resultantes.

De tal manera que sustituyendo (9) y (10) en (11) y (12), respectivamente, cada empresa maximiza sus utilidades y se obtienen los siguientes precios de equilibrio:

$$
\begin{aligned}
& p_{i}^{*}=\frac{q_{i}+v\left(x_{i}^{e}\right)-q_{\neg i}-v\left(x_{\neg i}^{e}\right)+p_{\neg i}+t+c_{i}}{2} \\
& p_{\neg i}^{*}=\frac{q_{\neg i}-v\left(x_{i}^{e}\right)-q_{i}+v\left(x_{\neg i}^{e}\right)+p_{i}+t+c_{i}}{2}
\end{aligned}
$$

Sustituyendo los valores de (13) y (14) en (11) y (12), respectivamente, tenemos que las utilidades de equilibrio resultantes son las siguientes:

${ }^{41}$ Esto es, costos fijos y costos variables. 


$$
\begin{aligned}
\pi_{i}^{*} & =\left[\frac{\left(q_{i}+v\left(x_{i}^{e}\right)-q_{\neg i}-v\left(x_{\neg i}^{e}\right)+p_{\neg i}+t-c_{i}\right)^{2}}{8 t}\right] \\
\pi_{\neg i}^{*} & =\left[\frac{\left(q_{\neg i}-v\left(x_{i}^{e}\right)-q_{i}+v\left(x_{\neg i}^{e}\right)+p_{i}+t-c_{\neg i}\right)^{2}}{8 t}\right]
\end{aligned}
$$

\section{ANÁLISIS DE RESULTADOS}

Con base en los resultados obtenidos realizaremos un análisis a lo largo del presente capítulo. Específicamente, se analizará la relación que guardan las variables de cobertura, precios y las utilidades de equilibrio respecto al resto de las variables que conforman los precios hedónicos de las empresas.

El interés principal de estudiar estas relaciones va encaminado a entender el proceso de elección de los consumidores entre dos opciones presentes en el mercado. Es decir, la influencia que ejerce sobre tal decisión cada una de las diferentes variables que conforman la propuesta de valor de cada empresa. Por otro lado, también se analiza el impacto que ejercen tales variables sobre el nivel de cobertura o participación de mercado de las empresas, así como sobre las utilidades obtenidas por cada empresa, una vez repartido el mercado.

Para este efecto, en la siguiente tabla se presenta el concentrado de estática comparativa, obtenida de los resultados del proceso de maximización y resolución del modelo.

En la Tabla 1 podemos observar que las variables de calidad del producto, $q_{i}$ y $q_{\neg i}$, presentan una relación positiva con el nivel de participación del mercado de su respectiva empresa. Sin embargo, un aumento en la calidad del 
producto estará negativamente relacionado con el nivel de participación de mercado de la empresa rival.

\section{Tabla 1}

Estática comparativa

\begin{tabular}{|l|l|l|l|l|}
\hline VARIABLES & $\lambda$ & $1-\lambda$ & $\pi_{i}^{e}$ & $\pi_{\neg i}^{e}$ \\
\hline$q_{i}$ & + & - & $+/-$ & $+/-$ \\
\hline$q_{\neg i}$ & - & + & $+/-$ & $+/-$ \\
\hline$p_{i}$ & - & + & $+/-$ & $+/-$ \\
\hline$p_{\neg i}$ & + & - & $+/-$ & $+/-$ \\
\hline$v\left(x_{i}^{e}\right)$ & + & - & $+/-$ & $+/-$ \\
\hline$v\left(x_{\neg i}^{e}\right)$ & - & + & $+/-$ & $+/-$ \\
\hline
\end{tabular}

Fuente: Elaboración propia.

Por otro lado, la relación de los precios de los productos, $p_{i}$ y $p_{\neg i}$, respecto al nivel de cobertura, es inversa respecto a la misma empresa y positiva respecto al nivel de cobertura de la empresa competidora.

Cuando se presenta un aumento en la percepción del valor de la red de una marca, éste provoca un aumento en el nivel de participación de mercado de la marca que lo generó y, por otro lado, provoca la disminución correspondiente del nivel de participación de la empresa competidora. Recordando que 
$v\left(x_{i}^{e}\right)=\delta x_{i}^{e}$ y que $x_{i}^{e}$ está formada por $z_{i}$ y $x_{i}$, entonces un aumento en el valor de se derivará del aumento en alguna de las variables que la conforman. Por un lado, un aumento en la percepción $\delta$, que es una variable exógena, podría provocar el aumento en el valor de . Por otro lado, el aumento puede provenir de considerar un mayor número de consumidores promedio por sucursal de la marca. Y por último, los resultados obtenidos sugieren que, un aumento en el número de sucursales de una marca podrá generar un aumento positivo sobre el tamaño de red de la marca y, por lo tanto, un impacto positivo sobre el nivel de participación de mercado de la empresa.

Con base en los resultados mostrados en la tabla anterior, podemos observar que para la totalidad de las variables bajo análisis, los efectos que ejercen sobre las utilidades de las empresas son ambiguos, es decir, pueden ser tanto positivos como negativos. El efecto final sobre las utilidades dependerá de la combinación de valores de las variables que los conforman. En el siguiente capítulo se presentarán los análisis de los resultados obtenidos bajo diversos escenarios, lo que ayudará a concluir bajo qué condicio-

$v\left(x_{i}^{e}\right) \quad$ nes de competencia las utilidades de las empresas se verán afectadas positiva o negativamente.

\section{ESCENARIOS DE COMPETENCIA ENTRE EMPRESAS}

A lo largo de esta parte, se presentarán diferentes posibles escenarios de competencia entre dos empresas. Tales escenarios se originaron otorgando diversos valores a cada una de las variables que conforman el nivel de participación de mercado y de las utilidades de las empresas.

Específicamente, analizaremos el comportamiento de la relación que guardan las variables $q_{i}, q_{\neg i}, p_{i}, p_{\neg i}, v\left(x_{i}^{e}\right), v\left(x_{\neg i}^{e}\right)$ y $c_{i}$ con el nivel de participación de mercado $\lambda$ y con las utilidades $\pi_{i}^{e}$.

La construcción de los escenarios se realizó de la siguiente manera. Para cada una de las variables antes mencionadas se consideraron tres valores diferentes: bajo, medio y alto. Con base en esto, se asignó a cada empresa 
diferentes combinaciones de valores para sus respectivas variables, dando para cada caso circunstancias de competencia particulares. De tal manera que, cada escenario supone diferentes valores para las variables de la empresa $i$ y de la empresa $\neg i$. Así, la construcción de un escenario supondrá que el valor de las variables de la empresa bajo análisis, así como de la competidora, se mantienen constantes, variando únicamente los valores de la variable que se quiera aislar para analizar su comportamiento. Es decir, una variable se analizará otorgándole los tres diferentes valores (bajo, medio y alto), ceteris paribus.

Este análisis se repite para cada una de las variables de la empresa analizada. Siguiendo este proceso, se realizaron nueve combinaciones de escenarios diferentes.

Esta construcción de escenarios, donde suponemos que todos los valores, excepto el de una variable, se mantienen igual, no excluye la posibilidad de que más de una variable pueda cambiar de valores en un mismo análisis, sin embargo, para efectos de simplificación no se contempla su análisis en el presente estudio.

Cabe mencionar que, bajo todos los escenarios considerados, se mantiene la misma relación de variables respecto a , presentada en la Tabla 1.

Para el caso de los precios de equilibrio, en la Tabla 2 se muestran las relaciones entre las variables que se obtuvieron para todos los escenarios.

Un aumento en el valor de la calidad de los productos, en el valor de la percepción sobre el tamaño de red o en los costos de una empresa, así como en el precio de la compañía competidora, generará un aumento en el precio de equilibrio de la empresa. Por otro lado, un aumento en la calidad de los productos y en la percepción del tamaño de red de la compañía rival generará que el precio de la empresa disminuya.

La Tabla 3 resume las relaciones entre las variables respecto a las utilidades de equilibrio, resultantes de los diversos escenarios de competencia.

A continuación se presentarán los resultados alcanzados más relevantes de los distintos escenarios, enfocándonos a los resultados relacionados con el efecto sobre las utilidades de la empresa. 
Externalidades de Red y su Influencia en la Percepción de Mercado

TABLA 2

Relaciones de variables respecto a los $p_{i}^{*}$

\begin{tabular}{|l|l|}
\hline VARIABLES & $p_{i}^{*}$ \\
\hline$q_{i}$ & + \\
\hline$q_{\neg i}$ & - \\
\hline$p_{\neg i}$ & + \\
\hline$v\left(x_{i}^{e}\right)$ & + \\
\hline$v\left(x_{\neg i}^{e}\right)$ & - \\
\hline$c_{i}$ & + \\
\hline
\end{tabular}

Fuente: Elaboración propia.

\section{Escenario 1: $i=$ bajo, = bajo}

Bajo esta combinación de valores se obtuvo que, la relación que guardan las utilidades respecto a las variables analizadas son en todos los casos positivas, inclusive cuando aumenta el valor de la calidad o de la red de la empresa competidora. Es decir, cuando tenemos valores bajos en todas las variables de las dos empresas y únicamente aumenta el valor de una de las variables de la competencia (p. ej. si aumenta el nivel de valoración de su red), el impacto negativo que pudiera tener sobre las utilidades de la empresa no es lo suficientemente fuerte como para generar una disminución sobre los mismos.

Por otro lado, un aumento en los costos de producción se compensa con el respectivo aumento en el precio, lo que hace que las utilidades presenten un comportamiento ascendente ante esta situación. 
TABLA 3

Relaciones de variables respecto a los

\begin{tabular}{|l|l|l|l|l|l|l|}
\hline Escenarios & $q_{i}$ & $q_{\neg i}$ & $p_{\neg i}$ & $v\left(x_{i}^{e}\right)$ & $v\left(x_{\neg i}^{e}\right)$ & $c_{i}$ \\
\hline $\mathrm{i}=$ bajo, -i= bajo & + & + & + & + & + & + \\
\hline $\mathrm{i}=$ medio, -i= bajo & + & $-/+$ & + & + & $-/+$ & - \\
\hline $\mathrm{i}=$ alto, -i= bajo & + & - & + & + & - & - \\
\hline $\mathrm{i}=$ bajo, -i= medio & $-/+$ & + & - & $-/+$ & + & $-/+$ \\
\hline $\mathrm{i}=$ medio, -i=medio & $-/+$ & $-/+$ & $-/+$ & $-/+$ & $-/+$ & $-/+$ \\
\hline $\mathrm{i}=$ alto, $-\mathrm{i}=$ medio & $-/+$ & - & + & $-/+$ & - & - \\
\hline $\mathrm{i}=$ bajo, $-\mathrm{i}=$ alto & - & + & - & - & + & + \\
\hline $\mathrm{i}=$ medio, -i= alto & - & $-/+$ & - & - & $-/+$ & + \\
\hline $\mathrm{i}=$ alto, -i= alto & - & - & - & - & - & - \\
\hline
\end{tabular}

Fuente: Elaboración propia.

Escenario 2: $i=$ medio, = bajo

Bajo este escenario, las utilidades de la empresa mantienen una relación positiva respecto a la calidad y la valoración de la red, así como con el precio de la empresa rival.

Sin embargo, el comportamiento de las utilidades presentó una conducta interesante respecto al aumento en la calidad de la competencia. Si la calidad del competidor cambia de baja a media ceteris paribus, las utilidades de la 
empresa disminuyen; sin embargo, al pasar de un valor medio a alto las utilidades vuelven a incrementarse pero a un nivel menor del que se encontraban cuando la calidad era baja. Adicionalmente, cuando aumenta el valor en la valoración de la red de la competencia las utilidades tienen el mismo comportamiento.

En este caso, un aumento en los costos de producción no podrá compensarse completamente con el aumento en el precio, y por lo tanto las utilidades disminuirán.

Escenario 3: $i=$ alto, $\neg i=$ bajo

Bajo estas condiciones, las utilidades mantuvieron un comportamiento equivalente al que tiene la cobertura con las variables. Es decir, las utilidades aumentarán cuando la calidad, la valoración de la red y el precio de la competencia aumenten, y disminuirá cuando la calidad y valoración de la competencia, así como los costos de producción, aumenten.

Al igual que en el escenario anterior, un aumento en los costos de produc-

$\neg i \quad$ ción, ceteris paribus, ocasiona un aumento en los precios que no compensa el aumento en los costos, provocando una disminución en las utilidades.

Escenario 4: $i$ = bajo, = medio

En este escenario, las utilidades tuvieron un comportamiento particular respecto a las variables de calidad y de valoración de la red de la empresa analizada. Al aumentar el valor de las variables mencionadas, de bajo a medio ceteris paribus, el valor de las utilidades disminuyó. Sin embargo, cuando vuelve a aumentar el valor de la calidad y de la red de medio a alto, las utilidades crecen alcanzando un nivel superior al que se encontraban cuando el valor de las variables era bajo.

Este comportamiento se puede explicar debido a que la empresa competidora tiene un nivel "medio" en la calidad, precio, valoración de la red y costos, por lo tanto cuenta con una clara ventaja en todas las variables a excepción del precio, la cual constituirá la única herramienta de la empresa para competir. Por lo tanto, al mantener todos los valores bajos, excepto el de calidad, su 
aumento tiene que ser significativo para que pueda repercutir en las utilidades de la empresa, de otra manera la empresa competidora estará en clara ventaja competitiva y los consumidores la preferirán. De igual manera, ante una desventaja competitiva, el aumento en la valoración de la red de la empresa tiene que ser significativo para que éste tenga repercusiones positivas sobre sus utilidades.

Por otro lado, ante un aumento en el nivel de calidad y del valor de la red de la empresa en competencia ceteris paribus, las utilidades de la empresa analizada, en lugar de disminuir presentan un comportamiento ascendente. Estos resultados resultan contra intuitivos.

Por último, un aumento en los costos de producción provocará, en primera instancia, una disminución en las utilidades, sin embargo cuando éstos aumentan su valor de medio a alto los precios mantienen su comportamiento ascendente de tal manera que, en este nivel, el aumento en los precios podrá compensar más que proporcionalmente el aumento en los costos, y por lo tanto las utilidades de la empresa se incrementarán.

Escenario 5: $i=$ medio, = medio

Bajo esta configuración de valores, las utilidades mantienen un comportamiento ambiguo respecto a todas las variables. En todos los casos, un aumento en el valor de la variable ocasionará una disminución en las utilidades, sin embargo cuando el valor aumenta hasta el nivel alto las utilidades entonces aumentarán.

Este caso representa un nivel de competencia alto. Partiendo de una situación en la que ninguna empresa tiene ventaja competitiva sobre la otra y, considerando que sus variables mantienen un valor medio, es difícil poder oscilar la balanza de preferencia o de participación de mercado hacia alguna de las dos. Para poder cambiar la proporción del mercado reflejada en sus utilidades, la empresa tendrá que aumentar sus variables de valor a un nivel muy superior respecto al de la competencia. Por otro lado, un aumento en las variables de valor de la competencia generará una disminución en las utilidades de la empresa, sin embargo, si continúan aumentando el efecto se revierte y ocasionan que la empresa aumente sus utilidades, lo cual resulta contra intuitivo. 
Por último, un aumento en los costos de producción provocará una disminución en las utilidades, al pasar de un valor bajo a medio, sin embargo, cuando éstos pasan de medio a alto las utilidades crecen puesto que los precios también continúan aumentando.

\section{Escenario 6: $i=$ alto, $\quad=$ medio}

Bajo este escenario, ante una ligera ventaja competitiva de la empresa respecto a la de su rival, la cual únicamente puede competir en precio, las relaciones de las variables con los beneficios muestran que el precio es más fuerte que las variables de valor ofrecidas por la empresa. Es decir, ante un aumento en la calidad y en la valoración de la red de la competencia, manteniendo sus precios más bajos que los de la empresa, generarán que las utilidades de ésta disminuyan, traduciéndose en una preferencia sobre los productos de la empresa rival. Adicionalmente, un incremento en las variables de valor de calidad y valoración de la red, para generar un aumento en las utilidades de la empresa, tienen que aumentar de bajo a alto, de otro modo el precio menor ofrecido $\neg i$ por la competencia, en combinación con los valores medios de sus variables, seguirá dominando las decisiones de compra de los consumidores.

Sin embargo, si el precio de la competencia aumenta, entonces las utilidades de la empresa se verán favorecidas, ya que sus mayores proposiciones de valor a los consumidores ejercerán su poder y generarán un efecto positivo en las utilidades al no tener en contra el precio menor de la empresa rival.

Por último, un aumento en los costos de producción ocasionará una disminución en las utilidades, la cual no se compensará por el aumento en los precios.

Escenario 7: $i=$ bajo, $\quad=$ alto

Bajo estas características de competencia, la empresa se encuentra en clara desventaja en todos los aspectos respecto a su rival. Su única proposición de valor representa el precio bajo, sin embargo no le es suficiente para compensar la diferencia tan grande respecto a la competencia en cuanto a proposiciones de valor a los consumidores. 
En este caso, un aumento en la calidad y en la valoración de la red, combinado con un precio bajo, no será suficiente como para competir con los valores tan elevados de la otra empresa, y por lo tanto sus utilidades disminuirán. Es decir, bajo estas circunstancias no será suficiente aumentar el valor de una variable si el valor de todas las demás es mayor para la competencia. Por otro lado, las utilidades de la empresa se incrementarán cuando la calidad y la valoración de la red de la empresa en competencia aumenten, lo cual resulta contra intuitivo.

Por último, un aumento en los costos de producción ocasionará un aumento tanto en los precios como en las utilidades. En este caso el aumento en los precios, más que compensar el aumento en los costos, genera un incremento en las utilidades de la empresa.

Escenario 8: $i=$ medio, = alto

En este escenario se sigue manteniendo una ligera ventaja competitiva de la empresa rival y, por lo tanto, se presentan efectos negativos sobre las utilidades respecto de las variables de calidad, valoración de red y precio de la competencia. Éste último efecto potencializa las ventajas competitivas de la competencia, ya que la única fortaleza con la que cuenta la empresa es la de un precio ligeramente menor. Por lo tanto este resultado nos indica que, aunque la competencia aumente sus precios, la empresa perderá utilidades ya que los consumidores valoran más aquellos atributos que les ofrece la competencia, y que sobrepasan las ventajas derivadas del menor precio que ofrece la empresa.

Adicionalmente, un aumento en la calidad y valoración en la percepción de la red de la empresa en competencia genera, en primera instancia, una disminución de las utilidades de la empresa, pero cuando toman un valor alto la relación con las utilidades se torna positiva, lo cual resulta contra intuitivo.

Un aumento en costos de producción generará un aumento tanto en precios como en las utilidades obtenidas, ya que los precios compensarán el aumento en los costos. 
Escenario 9: $i=$ alto, $\quad=$ alto

Este escenario representa el nivel de competencia más alto, en el cual todas las variables de valor son altas, es decir, ambas empresas tienen niveles altos de calidad, valoración de la red, precios y costos de producción.

Para estas características de competencia los beneficios guardan una relación negativa respecto a la totalidad de las variables. Lo que puede interpretarse como un mercado limitado y cerrado debido a los altos precios y estándares de calidad que manejan ambas empresas. Bajo estas circunstancias, la relación negativa respecto a las variables de calidad y valoración de la red de la empresa en competencia se conservará y, adicionalmente, un aumento en la calidad y en la valoración de la red de la empresa también ocasionará una disminución de las utilidades. Tal comportamiento pudiera explicarse debido a que los valores de las variables de ambas empresas se encuentran en la cumbre, por lo tanto, ningún valor puede aumentar el nivel de utilidades de la empresa. De tal manera que, cambiar los valores de cualquier variable tendría un efecto negativo sobre las utilidades de la empresa.

\section{CONCLUSIONES}

En el presente trabajo desarrollamos un modelo de localización de dos periodos, en el cual se considera la existencia de externalidades positivas de red en el consumo. Tomando el mercado con una densidad igual a uno, dos empresas compiten por los consumidores medidos como la participación de mercado. Una vez resuelto el modelo se supusieron diversos escenarios de competencia, mediante los cuales fue posible medir el efecto que tienen las externalidades de red sobre las utilidades de la empresa.

A su vez, los consumidores deciden qué marca consumir con base en los valores netos ofrecidos por las empresas, representados por las variables de calidad y valoración de la red, menos los precios pagados y los costos de transporte, inversamente relacionados con el nivel de cobertura de la empresa. 
Nuestro análisis revela que la cobertura de mercado de una empresa, medida como la participación de mercado, guarda una relación directa con la valoración del tamaño de la red por parte de los consumidores, al igual que con la calidad del producto y con en el precio de la empresa competidora. Por otro lado, el nivel de participación de mercado está inversamente relacionado con la calidad del producto, así como con el nivel de valoración de la red de la empresa rival y con el precio cobrado por la empresa. Estas relaciones se mantienen constantes bajo los nueve diferentes escenarios analizados, sin embargo el efecto sobre las utilidades no es contante. Este puede cambiar conforme cambian las circunstancias de competencia.

Con base en nuestro análisis de escenarios podemos concluir que, a medida que aumenta el valor de las variables el nivel de competencia aumenta también, lo que se traduce en menores utilidades para la empresa.

Por otro lado, cuando las empresas se encuentran bajo circunstancias de competencia similares, la relación de todas las variables, tanto de la empresa como de la empresa rival, presentan el mismo comportamiento respecto a las utilidades. Es decir, causan el mismo efecto tanto un aumento en la valoración de la red de la competencia como un aumento en la valoración de la red de la empresa.

Cuando se presentan valores medios de la empresa y de la empresa rival, el patrón de comportamiento de las utilidades es ambiguo respecto a algunas variables. Esto puede atribuirse a que la ventaja competitiva que tiene una empresa sobre la otra es muy pequeña, por lo tanto un cambio en la combinación de los valores puede nivelar la balanza hacia cualquiera de las dos empresas, siempre y cuando alcance el valor necesario para superar la ventaja que presenta la otra empresa.

El caso más significativo de estos escenarios es aquél donde ambas empresas mantienen valores medios, bajo el cual las relaciones de las variables respecto a las utilidades son negativas y sólo cuando una aumenta significativamente su valor el efecto sobre las utilidades es positivo. Esto nos indica que, únicamente un aumento extraordinario en la percepción de la red de la empresa (o de cualquier otra variable) significará un incremento en las utilidades. 
Por otro lado los resultados del modelo nos permiten concluir que, cuando existe una ventaja competitiva muy clara, entendida como valores altos de las variables consideradas contra valores bajos de la empresa contraria, entonces las relaciones de las variables respecto a las utilidades tendrán un comportamiento similar al que se obtuvo para el nivel de participación de mercado. Es decir, bajo estas circunstancias las utilidades de la empresa que tenga la ventaja competitiva aumentarán conforme aumente la calidad del producto, la valoración de su red y el precio de la competencia. Y, por otro lado, disminuirán a

medida que aumente la valoración de la red de la competencia o su calidad, o los costos de producción en los que se incurre.

De esta manera concluimos que, bajo la presencia de externalidades de red, el impacto de éstas sobre las utilidades de las empresas dependerá de las circunstancias de la competencia bajo las que se desenvuelvan. Adicionalmente, podemos concluir que cuando existe una clara ventaja competitiva de una empresa respecto a otra, en cuanto al valor de la valoración de la red, es difícil cambiar esta estructura y, en la mayoría de los casos, los consumidores preferirán consumir aquélla que les ofrezca una mayor oferta de valor, aunque esto signifique pagar un precio más alto.

\section{BIBLIOGRAFÍA}

Bensen, Stanley M. (1999), "Innovation, Competition, and the Theory of Network Externalities", presented at the Yale University Department of Economics Reunion, The World Economy in the 21 ${ }^{\text {st }}$ Century, 1999.

Bensen, Stanley M. y Joseph Farrell (1994), "Choosing How to Compete: Strategies and Tactics in Standardization”, Journal of Economic Perspectives, Vol. 8, No. 2, pp. 117-131.

Bental, Benjamin y Menahem Spiegel (1995), "Network Competition, Product Quality, and Market Coverage in the Presence of Network Externalities", Journal of Industrial Economics, Vol. 43, No. 2, pp. 197-208.

Corporativo Fragua, Informe Annual 2007. 
Davis, Scott M. (2002), LA MARCA máximo valor de su empresa, Prentice Hall.

Economides, Nicholas (1996), "The economics of networks", International Journal of Industrial Organization, Vol. 14, No. 2, pp. 673-699.

Economides, Nicholas y Frederick Flyer (1997), "Compatibility and Market Structure for Network Goods”, Working Papers 98-02, New York University, Leonard N. Stern School of Business Department of Economics.

Farrell, Joseph y Garth Saloner (1986), "Instaled Base and Compatibility: Innovation, Product Preannouncements, and Predation", The American Economic Review, Vol. 76, No. 5, pp. 940-955.

Femsa, Informe Annual 2007.

Grupo Santander, Informe Anual 2007.

Henard, David H. (1998), "Network externalities: The phenomenon of increasing returns and opportunities for strategy research", American Marketing Association. Conference Proceedings, pp. 385-391.

Homs, R. (2003), La era de las marcas depredadoras, Mc. Graw Hill.

HSBC Holding pcl, 2007 Annual Report and Accounts.

Inditex, Reporte Anual 2007.

Katz, Michael y Carl Shapiro (1985), "Network Externalities, Competition, and Compatibility", The American Economic Review, Vol. 75, No. 3, pp. 424440.

Katz, Michael y Carl Shapiro (1992), "Product Introduction with Network Externalities", The Journal of Industrial Economics, Vol. 40, No. 1, pp. 55-83.

Kotler, Philip y Gary Armstrong (2003), Fundamentos del Marketing, PearsonPrentice Hall.

Kotler, Philip, Dipak C. Jain y Suvit Maesincee (2002), El marketing se mueve. Una nueva aproximación a los beneficios, el crecimiento y la renovación, Paidós.

Kotler, Philip y Kevin Keller (2006), Dirección de Marketing, Pearson-Prentice Hall.

Leibenstein, H. (1950), "Bandwagon, Snob, and Veblen Effects in the Theory of Consumer's Demand", The Quarterly Journal of Economics, Vol. 64, No. 2, pp. 183-207. 
Externalidades de Red y su Influencia en la Percepción de Mercado

Liebowitz, B.J. y Stephen E. Margolis, (1994), "Network externality: an uncommon tragedy", The Journal of Economic Perspectives, Vol. 8, No. 2, pp. 133150.

McDonald's Corporation 2007, Annual Report.

Montalvo, Raúl F. (2003), "Banking Competition and Mergers", Chapter 1, Ph.D. thesis, Universidad de Essex, Inglaterra.

Saloner, Garth y Andrea Shepard (1995), “Adoption of Technologies with Network Effects: An Empirical Examination of the Adoption of Automated Teller Machines", The RAND Journal of Economics, Vol. 26, No. 3, pp. 479-501.

Starbucks Fiscal 2007, Annual Report.

Starbucks Coffee Company Fiscal 2007, Third Quarter Financial Results Prepared Remarks.

Starbucks Corporation, 10-K Report, Fiscal 2007.

Tirole, Jean (2000), The Theory of Industrial Organization, MIT Press.

Xie, Jonhong y Marvin Sirbu (1995), "Price Competition and Compatibility in the Presence of Positive Demand Externalities", Management Science, Vol. 41, No. 5, pp. 909-926. 